\title{
Catatonia in autism: a distinct subtype?
}

\author{
M. Ghaziuddin, P. Quinlan \& N. Ghaziuddin
}

University of Michigan, Ann Arbor, MI, USA

\begin{abstract}
Catatonia is a life-threatening disorder characterized by motor abnormalities, mutism, and disturbances of behaviour, which is increasingly being diagnosed in persons with autism. In this report, we describe the presentation and course of catatonia in an adolescent with autism who responded to electroconvulsive therapy (ECT). The illness started with depressive symptoms, but the predominant feature was one of extreme obsessive slowing and immobility. We propose that catatonia should be ruled out as a cause of regression sometimes seen in adolescents with autism, and that catatonia of autism may index a distinct subtype with a particularly poor outcome.
\end{abstract}

Keywords Asperger syndrome, autism, catatonia, comorbidity, depression, ECT

\section{Introduction}

In I874, Kurt Kahlbaum, a German psychiatrist, described catatonia as a distinct disorder characterized by disturbances of behaviour and abnormalities of motor function (Fink 200I). According to the Diagnostic and Statistical Manual for Mental Disorders, Fourth Edition (DSM-IV) (American Psychiat-

Correspondence: Dr Mohammad Ghaziuddin, University of Michigan Medical Center, PO Box 0277, Ann Arbor, MI 481090727, USA (e-mail: mghaziud@umich.edu).

(C) 2005 Blackwell Publishing Ltd ric Association 1994), it is often associated with schizophrenia, mood disorders, and general medical conditions, such as drug withdrawal and liver failure. Its main features are immobility, extreme negativism, mutism, and peculiarities of voluntary movements; these are sometimes combined with echolalia and bursts of hyperactivity. Although its prevalence is said to be decreasing - possibly because of earlier diagnosis and treatment of psychotic disorders - it still occurs in about 10\% of acutely ill psychiatric patients. If untreated, it can cause death because of dehydration and renal failure.

Catatonia is being increasingly recognized in persons with autism, a disorder characterized by a distinct pattern of social and communication deficits with a restricted range of interests starting before 3 years of age. However, relatively little is known about the presentation and treatment of catatonia in autism. In a longitudinal study, Lockyer \& Rutter (I970) found that $12 \%$ of the subjects showed neurologic regression during adolescence marked by a loss of language skills, intertia and intellectual decline. In many cases, this was accompanied by seizures. Based on the symptoms described, it is possible that some of these subjects suffered from catatonia. In a more recent survey of 506 children and adults referred for a diagnostic evaluation for autism, Wing \& Shah (2000) found that 30 subjects met the operational criteria of catatonia adopted in the study. A further eight individuals displayed some symptoms of the disorder without meeting all the criteria. 
M. Ghaziuddin et al. - Catatonia in autism: a distinct subtype?

Majority of the patients had their onset of catatonia between 15 and 19 years of age. The authors did not give details about the psychiatric aspects of their cases, but found that those who were language impaired and those who were passive in their social interactions were more vulnerable. Details about the treatment were not described. Realmuto \& August (I99I) described catatonia in three cases of autism and argued that the presence of psychiatric disorders increased the risk of catatonia emerging in persons with autism. Zaw et al. (I999) described a I4-yearold youngster with autism and catatonia. He displayed symptoms of mutism, akinesia, posturing, and rigidity. In addition to the symptoms of catatonia, he appeared depressed and showed nonspecific psychotic symptoms. A course of electroconvulsive therapy (ECT) improved the condition dramatically, although the core symptoms of autism were not affected. Dossche (1998) described a I5-year-old high-functioning youngster with autism who developed catatonia in the setting of auditory hallucinations, waxy flexibility, posturing, and physical aggression. His mood was constricted. A diagnosis of schizophrenia was made, and the patient responded to a course of neuroleptics and benzodiazepines. In another follow-up study of adults with autism and autistic-like conditions, out of a cohort of 120 subjects, at least $\mathrm{I}_{3}$ had clinically diagnosed catatonia (E. Billstedt, personal communication; see also Gillberg \& Billstedt 2000).

We describe here a youngster with autism who presented with catatonia during adolescence. His symptoms started after puberty and did not respond to trials with psychotropic medications. ECT was administered with varying degrees of success. Consent for publication of was obtained from the parents and from the Institutional Review Board (IRB) of the University of Michigan, Ann Arbor, MI, USA.

\section{Case histories}

Case I

K.W. is a I7-year-old white male with a history of autistic disorder, recurrent depression, and mild ID. He was admitted to the adolescent inpatient unit because of progressive worsening of mood accompanied by a generalized slowing of movements. He had started looking increasingly sad and depressed, and had also become almost mute, speaking only in monosyllables. His food intake had decreased and he had also lost weight.

The family history was positive for depression. The patient's mother had depression. His pregnancy and birth were uncomplicated. However, all his milestones were delayed. He was diagnosed with autism and mild ID at about 4 years of age. He attended a programme for children with autism throughout his school years, and received social skills training and language intervention.

He had a 3-year history of progressive decline in his level of functioning. Parents recalled that he was functioning reasonably well till he reached his puberty. After this, he became increasingly depressed and slowed down over a period of 6 months. $\mathrm{He}$ needed to be prompted to initiate simple activities such as getting dressed and eating. His behaviour in the classroom also deteriorated. He would stand alone or sit for long periods, doing nothing. He would spend several hours in the bathroom, repeatedly washing his hands or looking at himself in the mirror. In addition, his parents observed many types of abnormal movements, such as eye-blinking, eyerolling with jerking of the neck.

Prior to the onset of his symptoms, he was described as a well-adjusted youngster with autism. He spoke spontaneously, and was able to carry on a simple conversation. In addition to English, he spoke and read Hebrew. His school reports described him as being in the mildly intellectually disabled range. His parents dated the onset of his symptoms to his trip to an overnight camp for I month. After returning from the camp, his behaviour started deteriorating. He seemed to show less facial expression; he appeared somewhat gaunt and his language became sparse. Over the next 4 months, he became progressively mute, with marked slowing of movements, a depressed mood, and a gradual loss of independence of his activities of daily living. He was no longer able to function at school. He also showed increased motor tics, consisting of frequent turning his head to the side, blinking rapidly and gazing rightward and upward. He had a battery of investigations to rule out any physical cause of the condition all of which were in the normal range.

A diagnosis of depression with obsessive compulsive features was made. Over the course of 6 months, the excessive slowing of his movements increased, 
M. Ghaziuddin et al. • Catatonia in autism: a distinct subtype?

resulting in his dropping out of school. The parents used the analogy of watching a movie in slow motion to describe his extreme slowness. He received several trials of antidepressants, all of which failed. A trial of the antidepressant Citaloprim produced little benefit. $\mathrm{He}$ was initially started on a dose of $20 \mathrm{mg}$ daily. He was maintained on it for about 6 months. Risperdal was subsequently added in the dose of I $\mathrm{mg}$ a day, but had to be discontinued because of excessive sedation. Other drugs that had been tried in the past included zyprexa and depakote (details of the doses were not available). Because of his excessive slowing, and his decline in self-care, he was diagnosed with catatonia and depression.

The patient was admitted and assessed, and recommendations were made after two consultations with independent child psychiatrists for the patient to undergo ECT. The major reason that justified a course of ECT was his weight loss and declining selfcare. He had lost about $30 \mathrm{lb}$ of weight, dropping to $89 \mathrm{~kg}$. The treatment was administered after taking all ethical and medical issues into consideration as required by the practice parameters for ECT laid down in the Practice Parameters of the American Academy of Child and Adolescent Psychiatry (Ghaziuddin et al. 2004). Briefly, he was assessed by two independent child psychiatrists who agreed with the treatment. A third board certified psychiatrist with experience in ECT then assessed the patient.

Informed and written consent was obtained from the parents. He was assessed by a multidisciplinary team consisting of psychiatrists, nurses, social workers, and educational consultants. In addition, he was screened for any organic and medical problems by a team consisting of an anaesthetist, a paediatric cardiologist, and a paediatric neurologist. Opthalmologic examination using a fundoscope and a slit-lamp did not reveal any Kayser-Fleisher rings of Wilson's Disease. An electrocardiogram (EKG) and a brain magnetic resonance imaging (MRI) were also carried out and were found to be normal. All the blood tests, including tests for thyroid function, were read as normal. He was given a course of I 8 ECTs which proved life-saving, in that, he became increasingly verbal, he started eating his food as before, and his movements became more spontaneous. He was maintained as an outpatient on antidepressant but he never regained his premorbid level of functioning.

\section{Discussion}

This case report describes the presentation of catatonia in an adolescent with autism and adds to the sparse literature on this topic. It underscores the possibility that catatonia may be a risk factor in the developmental course of autism and that regression in behaviour seen in some autistic adolescents may result from the emergence of catatonia. In addition, it suggests that a subtype of patients with autism spectrum disorders may permanently lose their previous level of functioning after the onset of catatonia.

The patient had been diagnosed with autism in his early childhood and was well known to the local services. He received services for children with autism, and was categorized as 'autistic impaired' in the school system. At presentation, however, despite the catatonia, he had features consistent with a diagnosis of autistic disorder. He had a history of severe weight loss, crying spells, loss of appetite, and a family history of depression. He presented with bradykinesia, immobility, and muteness. An increase in posturing was also apparent. This shows that chronic comorbid psychiatric illness, such as depression, may be a risk factor for the development of catatonia in this population.

The main reason for giving ECT was failure of psychotropic medications, and the risk posed to life because of immobilization and refusal to eat. ECT improved mobility and facilitated food intake. However, the patient did not reach his premorbid level of functioning. This shows that in some patients with catatonia and autism, the course may be marked by relapses and remissions.

While the diagnosis of catatonia was based on the DSM-IV criteria, it is important to accurately distinguish it from autism. Of the criteria given for the diagnosis of catatonia in the DSM-IV, at least two overlap with features often seen in autism. These are posturing, stereotypies, and alterations in the level of activity. However, the key issue in the diagnosis of catatonia in autism is the emergence of new symptoms or a change in the type and pattern of preexisting symptoms. This issue is of importance because not every patient with autism who shows posturing should be considered to have catatonia. On the other hand, any patient with autism who shows an increase in posturing or stereotypies or a decrease in movements (often the result of obsessive slowing) 
M. Ghaziuddin et al. • Catatonia in autism: a distinct subtype?

should be examined for the presence of catatonia. This is particularly relevant if the patient is known to suffer from a comorbid psychiatric disorder, such as depression or obsessive compulsive disorder (Ghaziuddin et al. 2002). Obsessive compulsive symptoms may be more common in the catatonia of autism than in the rest of the population.

In summary, catatonia may not be as uncommon in persons with autism as generally believed. Followup studies of autism have documented the occurrence of catatonia, although not much has been written about its treatment. While its cause is not clear, catatonia is often associated with comorbid psychiatric disorders, such as depression and obsessive compulsive disorder. Of note, marked obsessive slowing, seen in some persons with autism, may be a forerunner of catatonia. Also, at least in some cases, regression in behaviour that is seen after puberty may result from the emergence of catatonic symptoms. Although the usual cause of catatonia is depression, it is unclear if this is also the case in persons with autism and pervasive developmental disorders. It is conceivable that the catatonia of autism is a distinct subtype that is associated with a poor outcome. Timely diagnosis is important because of the risk of starvation and death. ECT can be life-saving in these circumstances.

\section{References}

American Psychiatric Association (1994) Diagnostic and Statistical Manual for Mental Disorders, Fourth Edition. Author, Washington DC.
Dossche D. (1998) Catatonia in autistic disorders. Fournal of Autism and Developmental Disorders 28, 329-31.

Fink M. (200I) Catatonia: syndrome or schizophrenia subtype? Recognition and treatment. Fournal of Neural Transmission 108, 637-44.

Ghaziuddin M., Ghaziuddin N. \& Greden J. (2002)

Depression in autism and related disorders: clinical and research implications. Fournal of Autism and Developmental Disorders 32, 299-306.

Ghaziuddin N., Kutcher S. P., Knapp P. \& Work Group on Quality Issues (2004) Summary of the practice parameter for the use of electroconvulsive therapy with adolescents. Fournal of the American Academy of Child and Adolescent Psychiatry 43, II9-22.

Gillberg C. \& Billstedt E. (2000) Autism and Asperger syndrome: coexistence with other clinical disorders. Acta Psychiatrica Scandinavica 102, 321-30.

Lockyer L. \& Rutter M. (I970) A five to fifteen-year followup study of infantile psychosis: patterns of cognitive ability. British fournal of Clinical and Social Psychology 9, I5263.

Realmuto G. M. \& August G. J. (I99I) Catatonia in autistic disorder: a form of comorbidity or variable expression. Fournal of Autism and Developmental Disorders 2I, 517-28.

Swedo S. E. (2002) Pediatric autoimmune neuropsychiatric disorders associated with streptococcal infections (PANDAS). Molecular Psychiatry 7 (Suppl. 2), S24-5.

Wing L. \& Shah A. (2000) Catatonia in autistic spectrum disorders. British Fournal of Psychiatry 176, 357-62.

Zaw F. K., Bates G. D., Murali V. \& Bentham P. (1999) Catatonia, autism, and ECT. Developmental Medicine and Child Neurology 4I, 843-5.

Accepted Io May 2004 